\title{
Melatonin as an adjuvant therapy in preterm infants with neonatal sepsis, randomized trial
}

\author{
Zeinab A. El-Kabbany ${ }^{1}$, Ola G. El-Farghali ${ }^{1}$, Soha M. Khafagy ${ }^{1}$, Hebatallah A. Shaaban ${ }^{1 *}$ (D) Heba H. A. Osman² and \\ Mohamed H. Metwally ${ }^{1}$
}

\begin{abstract}
Background: Neonatal sepsis (NS) is a systemic uncontrolled inflammatory response to an infectious agent, resulting in oxidative stress. Melatonin antioxidant and free radical scavenger proved to be safe in neonates. We aimed to evaluate the efficacy and safety of melatonin as adjuvant therapy in NS. This was an interventional trial conducted on 40 preterm neonates with NS diagnosed on the basis of clinical and laboratory criteria. They were simply randomized to melatonin treated (MT) $(n=20)$ and conventionally treated (CT) $(n=20)$ groups. Melatonin was administered at a total dose of $20 \mathrm{mg}$ enterally in two divided doses of $10 \mathrm{mg}$ each, $1 \mathrm{~h}$ apart.

Malondialdehyde (MDA) was assessed in patients at enrollment, 4 and $72 \mathrm{~h}$ later in MT, and 72 hours later in CT. Fifteen healthy matched neonates were included as a control.

Results: There were no significant differences between MT and $C T$ groups regarding baseline sepsis workup. However, total leucocytic count, absolute neutrophil count, and C-reactive protein were significantly higher and platelets were significantly lower in CT compared to MT after $72 \mathrm{~h}$. MDA was doubled in CT while reduced in MT $72 \mathrm{~h}$ after intervention $(p=0.000)$. Mortality was significantly lower in MT. No side effects following melatonin administration were reported.
\end{abstract}

Conclusion: Melatonin is effective and safe adjuvant for treatment of NS that improves clinical and laboratory outcomes. Trial registration: clinicalTrials.gov Identifier: NCT03295162. Registered 27 September 2017-Retrospectively registered.

Keywords: Melatonin, Neonatal sepsis, Oxidative stress, MDA

\section{Background}

Neonatal sepsis (NS) is a leading cause of neonatal morbidity and mortality and a major public health problem, especially in developing countries and particularly in preterm infants $[1,2]$. It constitutes a systemic inflammatory response against the invading pathogen, where both endothelial cells and neutrophils are activated to release oxygen-derived free radicals; the so-called oxidative stress, resulting in cellular injury and organ failure [3].

Healthy adults have well developed and integrated antioxidant defenses to oppose the potential negative action of reactive oxygen species (ROS). Newborns, especially the

\footnotetext{
*Correspondence: Heba_Ali@med.asu.edu.eg

'Department of Pediatrics, Faculty of Medicine, Ain Shams University,

Abbassia Square, P.O. Box 11381, Cairo, Egypt

Full list of author information is available at the end of the article
}

premature, on the other hand, are very susceptible to oxidative stress due to an imbalance between the enhanced ROS production and the deficient antioxidant ability $[4,5]$.

Melatonin is an endogenously produced indolamine in adult humans, but found only in minimal amounts in neonates; especially preterm infants who are melatonin deficient. It is synthesized in the pineal gland from the neurotransmitter serotonin. It has a variety of physiological functions, not only as chronobiotic, but also visual, reproductive, cerebrovascular, and neuroendocrine functions. It is also cytoprotective and enhances free radical scavenging $[4,6]$.

Melatonin was used in many diseases during the perinatal period, some research workers studied its beneficial effect on the free radical disease in the newborn, including asphyxia, respiratory distress syndrome, surgical processes, 
and sepsis [7]. Studies related to the safety of melatonin have not discovered evidence of toxicity in either animals or humans even when given in very high doses [8].

We hypothesized that melatonin can be used as adjuvant therapy in treating septic preterm infants. So, this randomized interventional study aimed at evaluation of the efficacy and safety of enterally administered melatonin in comparison to conventional sepsis treatment alone in preterm neonates, through measuring the levels of malondialdehyde acid (MDA) as a marker of oxidative stress ( primary outcome) and comparing other clinical and laboratory parameters (secondary outcome) of sepsis in both groups.

\section{Methods}

This was an interventional clinical trial conducted on 55 preterm neonates ( $<36$ weeks' gestation) admitted to the neonatal intensive care units (NICUs) of University Hospitals during the period from November 1, 2015 till July 31, 2017.

\section{Patients}

Forty preterm infants were diagnosed as having neonatal sepsis based on clinical signs and laboratory indicators including Rodwell's hematological score $\geq 3$ [9]. Fifteen healthy, growing, gender, and gestational age-matched preterm infants were studied as control after ruling out sepsis. These were included for the assessment of basal MDA levels in the healthy preterm.

A full history was taken for all neonates including maternal, obstetric, and perinatal history. Gestational age (GA) was calculated based on the date of the last menstrual period and confirmed by using the modified Ballard score [10].

Mode of delivery and Apgar score at 1 and 5 min were recorded. Birth anthropometric parameters were measured. All neonates received routine neonatal care according to our NICU protocol.

Preterm infants were excluded from the study if they had congenital anomalies, evidence of perinatal asphyxia, contraindication of enteral feeding, and/or oxygen needs with a fraction of inspired oxygen above $30 \%$, either on invasive or non-invasive mechanical ventilation.

The study design was a parallel trial with an allocation ratio of 1:1. Simple randomization of septic neonates $(n=$ 40) was done using odd- and even-numbered prewritten cards according to sequence of enrollment. We assigned odd numbers to melatonin treatment (MT group; $n=20$ ); combined with conventional therapy of sepsis, and even numbers to lone conventional therapy (CT group; $n=20$ ).

\section{Intervention}

Melatonin $10 \mathrm{mg}$ rapid release capsules (Puritans pride ${ }^{\circ}$ ) were used. MT group was given melatonin at a total dose of $20 \mathrm{mg}$ dissolved in $4 \mathrm{ml}$ of distilled water via the enteral route in two doses of $10 \mathrm{mg}(2 \mathrm{ml})$ each, with a 1-h interval

Laboratory investigations were done at the time of diagnosis of sepsis in patients and on day 1 in the control group and was repeated in patients on day 3 . These included C-reactive protein (CRP) assay by latex agglutination test and complete blood count using Sysmex XT-1800i (Sysmex, Kobe, Japan).

Venous samples were withdrawn in gel tubes and were allowed to clot for $30 \mathrm{~min}$ before centrifugation at $2000 \times g$ for 15 minutes at $4{ }^{\circ} \mathrm{C}$.

MDA detected by Colorimetric method using Lipid Peroxide (Malondialdehyde) Assay Kit supplied by BIODIAGNOSTIC ${ }^{\circledR}$ (cat no. MD 25 28). Serum MDA was reassessed in MT group 4 and $72 \mathrm{~h}$ after melatonin administration and in CT group, $72 \mathrm{~h}$ after the initial sample. Serum MDA concentrations were measured with an enzyme-linked immunosorbent assay (ELISA) kit according to the manufacturer's protocol (Biodiagnostics).

Blood cultures were done using BD BACTEC PEDS PLUS/F culture vials (Becton, Dickinson and Company Spark, Ireland).

\section{Sample size calculation}

Assuming an effect size for melatonin on CRP levels of one (1) a sample size of 20 would be enough to detect such effect if true at 0.05 alpha error and 0.8 power of the test.

\section{Statistical methods}

Data were collected, revised, coded, and entered into the Statistical Package for Social Science (IBM SPSS), version 23. Parametric quantitative data were presented as mean, standard deviation and range, while non-parametric data were presented as median and inter-quartile ranges (IQR). Qualitative variables were presented as numbers and percentages.

We used chi-square test and/or Fisher exact test for comparison of qualitative data within groups.

Independent $t$ test was used to compare parametric quantitative data and Mann-Whitney for non-parametric data

The comparison between two paired groups with quantitative data and parametric distribution was done by using paired $t$ test while data with non-parametric distribution were done by using Wilcoxon rank test. Spearman correlation coefficients were used to assess the correlation between two quantitative parameters in the same group.

The confidence interval was set to $95 \%$ and the margin of error accepted was set to $5 \%$. So, the $p$ value was considered significant if $<0.05$ and highly significant if $<0.01$. 


\section{Results}

Septic neonates were 26 males [65\%] and 14 females [35\%]. Control group included 10 males [66.7\%] and 5 females [33.3\%]. GA was $(32.40 \pm 2.60 ; 32.87 \pm 2.07$ weeks, respectively; $p=0.533)$.

There was no difference found between MT group and CT group regarding all neonatal and maternal demographic data; GA, birth weight, mode of delivery, and sex distribution $(p>0.05)$. Moreover, there were no significant differences between MT and CT groups regarding MAP, oxygen use, need for inotropic support, and need for anti-fungal therapy before melatonin treatment.

However, CT group had significantly longer hospital stay $5.5(3.5-9.5)$ vs. $3(2.5-4.5)$ days, $p=0.018)$ and significantly higher mortality $(30 \%$ vs. $0 ; p=0.008)$ than MT group.

Initial septic workup parameters were non-significantly different between the two septic groups; including total leucocytic count (TLC), absolute neutrophilic count (ANC), platelets count, CRP, and Rodwell's hematological score.

At $72 \mathrm{~h}$ after intervention, MT group showed a significant reduction of ANC and CRP, and significant increase of platelet count in comparison to initial levels $(p=$ 0.048, 0.000, and 0.020, respectively). Conversely, in CT group, TLC, ANC, and CRP were significantly elevated, while platelets significantly decreased $72 \mathrm{~h}$ after the basal values ( $p=0.002,0.003,0.003$, and 0.008 , respectively).

As regards MDA, initial values were significantly higher in MT group compared to control (5.19 \pm 0.07 vs. $1.36 \pm 1.60 ; p=0.000)$, and in CT group compared to control as well $(2.87 \pm 1.09$ vs. $1.36 \pm 1.60 ; p=0.002)$. After $72 \mathrm{~h}, \mathrm{MDA}$ in MT group was significantly lower than CT group $(2.68 \pm 0.06$ vs $5.18 \pm 1.93 ; p=0.000)$.

Within MT group neonates, MDA levels were significantly reduced after 6 and $72 \mathrm{~h}(p=0.000)$. Conversely, within CT group neonates, MDA levels were significantly increased after $72 \mathrm{~h}(p=0.000)$ as shown in (Fig. 1).

There was a significant percentage of change of different laboratory parameters, namely, TLC, ANC, platelets,
CRP, and MDA level between MT and CT groups (Table 1).

No side effects following melatonin administration have been reported using the present dose.

\section{Discussion}

Neonatal sepsis is a commonly encountered problem in NICUs, which is characterized by oxidative stress, release of cytokines, and mitochondrial dysfunction [11, 12]. In order to counteract free radicals damage, many strategies to augment antioxidant status in ill preterm infants have been described. Melatonin and its metabolites efficiently detoxify ROS and other free radicals, through the activation of several antioxidant mechanisms [4].

So, in this clinical trial, we administered enteral melatonin to a group of septic preterm neonates to test its efficacy in comparison to a group of matched neonates.

We used distilled water to dissolve the medicine. In the study of Gitto and colleagues [11], they used 1:90 mixture of ethanol/physiologic saline as a solvent for melatonin, which was not received by the septic infants not treated with melatonin. Although given in a very small amount, ethanol itself is reported to have antioxidative actions which can affect the results.

We documented that the serum inflammatory parameters, namely, TLC, ANC, platelet count, and CRP were similar before melatonin administration in all septic patients, however, TLC, ANC, and CRP were significantly higher and platelets count was significantly lower in CT group compared to MT group $72 \mathrm{~h}$ after basal values. The significant improvement in septic laboratory parameters, that we found, has been previously shown in other studies [11, 13]. In their study, Gitto et al. [11] documented a significant improvement both clinically and laboratory after $24 \mathrm{~h}$ in those neonates who received melatonin. This effect is predominantly related to the anti-inflammatory ability of melatonin.

Free radical and reactive species generated during sepsis induce lipid peroxidation of cell membranes which leads to increased serum levels of MDA [14]. In the present study, the significant reduction of MDA to less

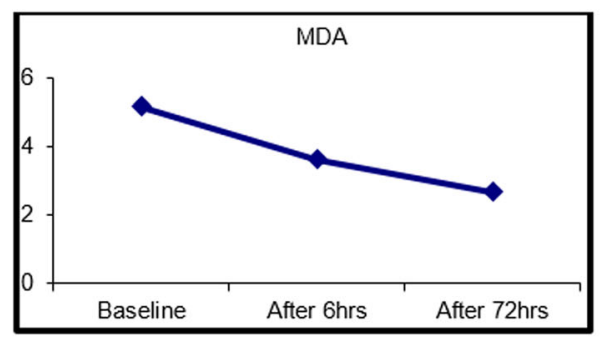

(A)

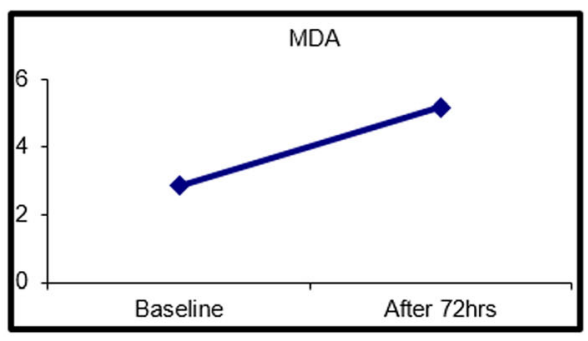

(B)

Fig. 1 MDA mean levels in (a) MT group (baseline, after 6 h, and after 72 h) and (b) CT group (baseline and after 72 h) 
Table 1 Comparison of the percentage of change of different laboratory parameters between MT and CT groups

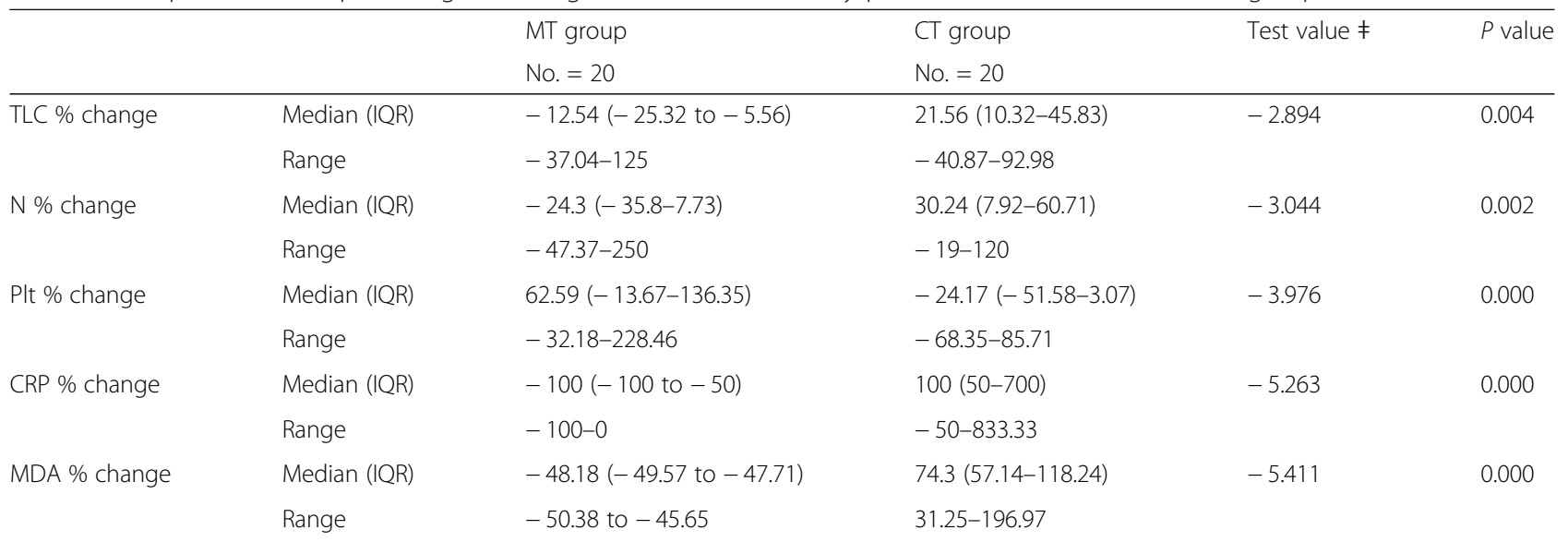

CRP C reactive protein, MDA malondialdehyde, $N$ neutrophilic count, $T L C$ total leucocytic count, Plt platelets

¥Mann-Whitney test

than half its initial levels after melatonin intake confirms the immediate melatonin antioxidant effect. Some human studies showed similar results with the return of lipid peroxidation products to normal levels $4 \mathrm{~h}$ after melatonin administration [11]. In animal models of sepsis, melatonin reduces oxidative damage, reduced MDA levels, improved mitochondrial function, and organ dysfunction and also decreases mortality [15-17].

As regards the clinical course of sepsis in the two septic groups in comparison to the control, there were no significant difference between control and MT group as regards mean arterial blood pressure (MAP) and inotropic use after melatonin treatment while CT group showed significantly less MAP, and more use of inotropes and more mortality than control group. This documents that melatonin use improved the clinical course of sepsis in these neonates.

Moreover, there was a shorter length of stay in the MT group compared to the CT group, and there was no mortality in the MT group while there were $6(30 \%)$ mortality in CT. This was comparable to $[11,13,18,19]$ who declared that as a therapeutic agent in sepsis, melatonin has been associated with decreased inflammatory markers and improved outcomes.

We reported no side effects following melatonin administration in our study. Many studies documented that short-term melatonin use is highly effective in reducing complications in the neonatal period with a remarkably benign safety profile, even with pharmacological doses $[11,20,21]$. None of the animal studies of perinatal melatonin exposure has shown any serious side effects, and no complications with long-term melatonin therapy in children and adults have been reported as well [22]. With the general agreement that melatonin therapy has low toxicity, even when children are treated with higher doses [8].
Limitations of our study are that we included both earlyonset sepsis and late-onset sepsis in both groups without subgrouping them in each of the studied groups, this was due to the small number of participants in each group.

\section{Conclusion}

Melatonin, being an antioxidant, offers perspectives of beneficial effects in neonatal sepsis with the improvement of clinical and laboratory parameters of inflammation and improve the clinical outcome. No adverse event has been observed in our population of newborns treated with melatonin.

\section{Abbreviations}

ANC: Absolute neutrophilic count; CT: Conventionally treated; CRP: C-reactive protein; GA: Gestational age; MAP: Mean arterial blood pressure;

MDA: Malondialdehyde; MT: Melatonin treated; NICU: Neonatal intensive care unit; NS: Neonatal sepsis; ROS: Reactive oxygen species; TLC: Total leucocytic count

\section{Acknowledgements}

Not applicable

\section{Authors' contributions}

ZE and OE conceptualized and designed the study. SK and HS contributed to the conceptualization and drafted the initial manuscript. $\mathrm{HO}$ and MM supervised data collection, laboratory investigations and analyzed and interpreted the data. All authors contributed to data interpretation and manuscript writing and have read and approved the final submission.

\section{Funding}

None

Availability of data and materials

The datasets used and/or analyzed during the current study are available from the corresponding author on reasonable request.

\section{Ethics approval and consent to participate}

Written consents were obtained from parents or caregivers of included neonates. The study protocol was approved by the Research Ethical Committee of Ain Shams University hospitals; ID: FMASUMD 250/2015 and it was designed to be in accordance with the Helsinki Declaration of 1975. This study is registered in clinicalTrials.gov (clinicalTrials.gov Identifier: NCT03295162). 


\section{Consent for publication}

Not applicable

\section{Competing interests}

The authors declare that they have no competing interests.

\section{Author details}

'Department of Pediatrics, Faculty of Medicine, Ain Shams University, Abbassia Square, P.O. Box 11381, Cairo, Egypt. ${ }^{2}$ Department of Clinical pathology, Faculty of Medicine, Ain Shams University, Abbassia Square, P.O. Box 11381, Cairo, Egypt.

Received: 26 June 2019 Accepted: 13 December 2019

Published online: 13 January 2020

\section{References}

1. Simonsen KA, Anderson-Berry AL, Delair SF, Davies HD (2014) Early-onset neonatal sepsis. Clin Microbiol Rev 27(1):21-47. https://doi.org/10.1128/CMR. 00031-13

2. Zea-Vera A, Ochoa TJ (2015) Challenges in the diagnosis and management of neonatal sepsis. J Trop Pediatr 61(1):1-13. https://doi.org/10.1093/tropej/ fmu079 Epub 2015 Jan 20

3. Narad SG, Gupta MH, Pakhmode SE (2013) Role of non-enzymatic antioxidants in neonatal septicemia. Int J Cur Tr Res 2(1):378-380

4. Gitto E, Marseglia L, Manti S et al (2013) Protective role of melatonin in neonatal diseases. Oxid Med and Cell Longev 2013:980374. https://doi.org/ $10.1155 / 2013 / 980374$

5. Ozsurekci Y, Aykac K (2016) Oxidative stress related diseases in newborns. Oxid Med Cell Longev 2016:2768365. https://doi.org/10.1155/2016/2768365

6. Aversa S, Marseglia L, Arco A et al (2012) 1640 Efficacy and safety of melatonin in neonates. Arch Dis Child 97:A464

7. Marseglia L, Manti S, D'Angelo G, Barberi I (2014) Melatonin for the newborn. J Pediatr Neonat Individual Med 3(2):e030232. https://doi.org/10. 7363/030232

8. Gitto E, D'Angelo G, Romeo C, Aversa S, Salpietro C, Reiter RJ (2012) Efficacy and safety of melatonin in newborn infants. Child J Pediatrics 1(1)

9. Rodwell RL, Leslie AL, Tudehope DI (1988) Early diagnosis of neonatal sepsis using a hematologic scoring system. J Pediatr 112:761-767

10. Ballard JL, Khoury JC, Wedig K, Wang L, Eilers-Walsman BL, Lipp R (1991) New Ballard Score, expanded to include extremely premature infants. J Pediatr 119(3):417-423

11. Gitto E, Karbownik M, Reiter RJ et al (2001) Effects of melatonin treatment in septic newborns. Pediatr Res 50(6):756-760

12. Galley HF, Lowes DA, Allen L, Cameron G, Aucott LS, Webster NR (2014) Melatonin as a potential therapy for sepsis: a phase I dose escalation study and an ex vivo whole blood model under conditions of sepsis. J Pineal Res 56(4):427-438. https://doi.org/10.1111/jpi.12134 Epub 2014 Apr 5

13. El Frargy M, El-sharkawy HM, Attia GF (2015) Use of melatonin as an adjuvant therapy in neonatal sepsis. J Neonatal Perinatal Med 8:227-232. https://doi.org/10.3233/NPM-15814072

14. Batra S, Kumar R, Kapoor AK, Ray G (2000) Alterations in antioxidant status during neonatal sepsis. Ann Trop Paediatr 20:27-33

15. Fink T, Glas M, Wolf A et al (2014) Melatonin receptors mediate improvements of survival in a model of polymicrobial sepsis. Crit Care Med 42(01):e22-e31

16. Lowes DA, Webster NR, Murphy MP, Galley HF (2013) Antioxidants that protect mitochondria reduce interleukin- 6 and oxidative stress, improve mitochondrial function, and reduce biochemical markers of organ dysfunction in a rat model of acute sepsis. Br J Anaesth 110(3):472-480. https://doi.org/10.1093/bja/aes577

17. Li Volti G, Musumeci T, Pignatello R et al (2012) Antioxidant potential of different melatonin-loaded nanomedicines in an experimental model of sepsis. Exp Biol Med (Maywood) 237(6):670-677

18. Srinivasan V, Pandi-Perumal SR, Spence DW, Kato H, Cardinali DP (2010) Melatonin in septic shock: some recent concepts. J Crit Care 25(4):656.e1-6. https://doi.org/10.1016/j.jcrc.2010.03.006

19. D'Angelo G, Marseglia L, Reiter RJ, Buonocore G, Gitto E (2017) Melatonin and neonatal sepsis: a promising antioxidant adjuvant agent. Am J Perinatol 34(14):1382-1388. https://doi.org/10.1055/s-0037-1604244 Epub 2017 Jul 13
20. Fulia F, Gitto E, Cuzzocrea S et al (2001) Increased levels of malondialdehyde and nitrite/nitrate in the blood of asphyxiated newborns: reduction by melatonin. J Pineal Res 31(04):343-349

21. Gitto E, Romeo C, Reiter RJ et al (2004) Melatonin reduces oxidative stress in surgical neonates. J Pediatr Surg 39(02):184-189

22. Sánchez-Barceló EJ, Mediavilla MD, Tan DX, Reiter RJ (2010) Clinical uses of melatonin: evaluation of human trials. Curr Med Chem 17(19):2070-2095

\section{Publisher's Note}

Springer Nature remains neutral with regard to jurisdictional claims in published maps and institutional affiliations.

\section{Submit your manuscript to a SpringerOpen ${ }^{\circ}$ journal and benefit from:}

- Convenient online submission

- Rigorous peer review

- Open access: articles freely available online

- High visibility within the field

- Retaining the copyright to your article

Submit your next manuscript at $\boldsymbol{\nabla}$ springeropen.com 